\title{
VOLUME CORPUSCULAR MÉDIO (VCM) E AMPLITUDE DA DISTRIBUIÇÃO DO TAMANHO DOS ERITRÓCITOS (RDW) EM EQUINOS DA RAÇA QUARTO DE MILHA USADOS EM PROVAS DE TRÊS TAMBORES
}

\author{
MEAN CORPUSCULAR VOLUME (MCV) AND RED BLOOD CELL \\ DISTRIBUTION WIDTH (RDW) IN QUARTER HORSES USED FOR \\ BARREL RACING
}

\author{
Renan Silva de Carvalho' \\ Lais Policarpo Macedo ${ }^{1}$ \\ Fernanda Almeida Teixeira ${ }^{1}$ \\ Marcela Bucher Binda ${ }^{1}$ \\ Clarisse Simões Coelho ${ }^{1 *}$
}

\author{
${ }_{1}^{1}$ Universidade de Vila Velha, Vila Velha, ES, Brasil. \\ *Autora para correspondência - clarisse.coelho@uvv.br
}

\begin{abstract}
Resumo

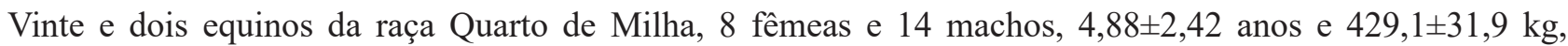
foram avaliados antes (T0) e imediatamente após (T1) e com 30 (T2) e 120 minutos (T3) após uma prova de três tambores para determinar a influência do exercício físico sobre o volume corpuscular médio (VCM) e a amplitude da distribuição do tamanho dos eritrócitos (RDW). Variáveis foram submetidas à análise de variância e a comparações entre médias pelo teste de Tukey e a comparações entre machos e fêmeas pelo teste-t, considerando-se $\mathrm{p}<0,05$. Os valores de VCM foram 46,5 $\pm 2,0 \mathrm{fl}, 47,6 \pm 2,2 \mathrm{fl}, 46,6 \pm 1,8 \mathrm{fl}$ e 46,7 $\pm 1,9 \mathrm{fl}$ em machos e 46,3 $\pm 0,6 \mathrm{fl}, 47,7 \pm 0,9 \mathrm{fl}, 46,7 \pm 0,8 \mathrm{fl}$ e 46,4 $\pm 0,8 \mathrm{fl}$ em fêmeas, respectivamente, em T0, T1, T2 e T3. Para RDW, foram registrados valores de $28,8 \pm 8,1 \%, 30,8 \pm 9,5 \%, 28,2 \pm 8,1 \%$ e $26,6 \pm 8,0 \%$ em machos e $28,0 \pm 7,3 \%, 27,1 \pm 8,1 \%, 28,1 \pm 8,1 \%$ e $28,6 \pm 7,2 \%$, em fêmeas, respectivamente, em T0, T1, T2 e T3. Houve aumento significativo somente de VCM nos equinos de ambos os sexos. Não foram observadas diferenças entre machos e fêmeas para VCM e RDW. Foi possível concluir que o exercício físico imposto levou a uma macrocitose homogênea.
\end{abstract}

Palavras-chave: cavalos; eritrócitos; exercício.

\begin{abstract}
Twenty-two Quarter Horses, eight females and 14 males, 4.88 \pm 2.42 years old and weighting $430.0 \pm 31.4 \mathrm{~kg}$, were evaluated before (T0), 5 minutes (T1), 30 minutes (T2), and 120 minutes (T3) after a barrel racing exercise to determine the influence of physical exercise on the mean corpuscular volume (MCV) and red blood cell distribution width (RDW). Variables were analyzed and comparisons were made using Tukey test, considering $\mathrm{p}<0.05$. Comparisons between males and females were done using t-test. Mean MCV values were $46.5 \pm 2.0 \mathrm{fl}$ for $\mathrm{T} 0,47.6 \pm 2.2 \mathrm{fl}$ for $\mathrm{T} 1,46.6 \pm 1.8 \mathrm{fl}$ for $\mathrm{T} 2$, and $46.7 \pm 1.9 \mathrm{fl}$ for $\mathrm{T} 3$ for males, and $46.3 \pm 0.6$ $\mathrm{fl}$ on $\mathrm{T} 0,47.7 \pm 0.9 \mathrm{fl}$ on $\mathrm{T} 1,46.7 \pm 0.8 \mathrm{fl}$ on $\mathrm{T} 2$, and $46.4 \pm 0.8 \mathrm{fl}$ on $\mathrm{T} 2$ for females. Mean RDW values were $28.8 \pm 8.1 \%$ for $\mathrm{T} 0,30.8 \pm 9.5 \%$ for $\mathrm{T} 1,28.2 \pm 8.1 \%$ for $\mathrm{T} 2$, and $26.6 \pm 8.0 \%$ for $\mathrm{T} 3$ for males, and $28.0 \pm$ $7.3 \%$ for $\mathrm{T} 0,27.1 \pm 8.1 \%$ for $\mathrm{T} 1,28.1 \pm 8.1 \%$ for $\mathrm{T} 2$, and $28.6 \pm 7.2 \%$ for T3 on females. A significant increase for MCV on both males and females was observed. No differences were observed between values recorded on males and females for MCV and RDW. It was possible to conclude the physical activity imposed on the present research leaded to a homogeneous macrocytosis.
\end{abstract}


Keywords: equines; erythrocytes; exercise.

Enviado em: 25 maio 2015

Aceito em: 12 maio 2016

\section{Introdução}

Variáveis hematimétricas podem ser usadas na avaliação dos efeitos do exercício e do treinamento físico ${ }^{(1,2)}$. Aumentos nos valores hematimétricos ocorrem de acordo com a intensidade do exercício e devem-se, principalmente, à contração esplênica esforço-dependente ${ }^{(3)}$. Segundo Hanzawa e Watanabe ${ }^{(4)}$, essa contração esplênica nos equinos é a mais sensível dentre as espécies animais, podendo levar a aumentos de até $50 \%$ na população eritrocitária. Esse efeito fisiológico é vantajoso, particularmente nos equinos atletas, pois eleva a capacidade aeróbia ao aumentar o transporte de oxigênio para a musculatura esquelética ativa ${ }^{(5)}$. Exercícios de intensidade submáxima geram menor atividade simpática e, consequentemente, uma contração esplênica parcial, do que os exercícios de alta intensidade ${ }^{(6)}$. McKeever et al. ${ }^{(7)}$ ressaltaram que o aumento do volume eritrocitário é importante até um certo limite, devido à intensa correlação entre aumento de volume globular (VG) e a viscosidade sanguínea, com consequente queda de performance.

Alguns autores descreveram que as alterações hematimétricas ocorrem também em consequência de outros fatores, além da contração esplênica. Com a realização da atividade física, há uma redução no volume plasmático devido às perdas pela sudorese / respiração, na tentativa de dissipar o calor produzido pela contração muscular, principalmente em condições de elevada temperatura ambiental e umidade relativa do ar, e à movimentação transitória dos fluidos entre os compartimentos extra e intracelular, devido às alterações na pressão sanguínea ${ }^{(7,8)}$. Muñoz et al. ${ }^{(9)}$ reforçaram que o exercício físico leva a alterações na hidratação e no conteúdo eletrolítico que, por sua vez, podem afetar as propriedades volumétricas dos eritrócitos.

O volume corpuscular médio (VCM) é o índice hematimétrico absoluto calculado pelo VG e pela contagem eritrocitária, sendo comumente usado para avaliar o grau de anisocitose ${ }^{(10)}$. Segundo citação de Balarin et al. ${ }^{(11)}$, seria necessário um percentual muito elevado de células com volume alterado para produzir um VCM anormal. Outra técnica para avaliar a anisocitose seria a avaliação morfológica dos eritrócitos, mas esse é um método muito subjetivo ${ }^{(10)}$. Os valores de referência descritos para VCM em equinos da raça Puro Sague Inglês (PSI) oscilam entre 41 e $49 \mathrm{fl}^{(12)}$.

O índice de amplitude de distribuição eritrocitária (RDW) reflete a heterogeneidade do tamanho dos eritrócitos, sendo o índice mais sensível para determinar o grau de anisocitose ${ }^{(11)}$. Segundo Easley ${ }^{(13)}$, em equinos, o RDW é importante na identificação de aumento na eritropoiese e na avaliação de quadros anêmicos, sendo os valores médios em animais clinicamente sadios de 15,2\% a 19,8\%. Radin et al. ${ }^{(14)}$ citaram valores de $24,3 \%$ para equinos com quadro de hemorragia grave e de $26,4 \%$ em equinos com quadro de hemólise intensa.

Os valores citados na literatura para VCM e RDW, tanto no repouso quanto após treinamentos e atividade física, são conflitantes. Balarin et al. ${ }^{(11)}$ descreveram valores de RDW para equinos da raça PSI saudáveis bem semelhantes aos descritos por Radin et al. ${ }^{(14)}$ para animais com grave hemorragia e/ou hemólise. Existem relatos descrevendo redução ${ }^{(15,16)}$, aumento ${ }^{(17,18)}$ e até a não variação dos valores $^{(10,19-21)}$ de VCM após atividade física. Similarmente, autores descrevem aumento ${ }^{(10,20)}$ ou não oscilação ${ }^{(21)}$ de RDW após diferentes exercícios físicos. Segundo Lippi et al. ${ }^{(22)}$, os resultados diversos e controversos ocorrem devido à heterogeneidade das amostras (atletas e não atletas), tipo de esporte (sprint, enduro ou misto), volume e intensidade do exercício (curto, médio ou longa duração). Melo et al. ${ }^{(2)}$, Muñoz et al. ${ }^{(9)}$ e Piccione et al. ${ }^{(23)}$ acrescentam, ainda, a influência de fatores relacionadas às raças usadas, sexo dos animais, localidade do experimento, momentos de coleta de sangue e procedimentos analíticos.

Segundo Ferraz et al. ${ }^{(18)}$, é essencial que pesquisas sejam feitas em condições tropicais para tornar viável o uso de tais variáveis fisiológicas na determinação da aptidão atlética de equinos. Além disso, 
a maioria dos estudos avaliou equinos da raça PSI ${ }^{(3,10,11,17)}$. Por isso, o propósito da presente pesquisa foi avaliar a influência do exercício físico (prova de três tambores) sobre VCM e RDW em equinos da raça Quarto de Milha.

\section{Material e Métodos}

O presente projeto de pesquisa teve aprovação do Comitê de Bioética Animal da Universidade Vila Velha (CEUA - UVV-ES), sendo registrado sob o número 318/2014.

Foram utilizados 22 equinos da raça Quarto de Milha, sendo oito fêmeas e 14 machos, pesando

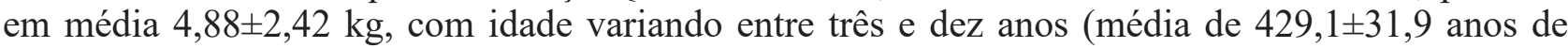
idade), considerados clinicamente hígidos, mediante exame clínico (exame físico e hemograma). Estes animais pertencem à criatórios de equinos Quarto de Milha, localizados nos municípios de Vila Velha e Guarapari, estado do Espírito Santo, Brasil. Todos os animais foram submetidos ao mesmo tipo de manejo alimentar e sanitário. A alimentação era feita com feno de coast-cross (Cynodon dactylon x Cynodon nlemfluensis) ad libitum e ração comercial, com $12 \%$ de proteína bruta, a 1\% do peso corporal, oferecidos três vezes ao dia. A água e sal mineral foram fornecidos ad libitum.

Todos os equinos selecionados encontravam-se no mesmo nível de condicionamento atlético e executavam tal atividade há pelo menos seis meses. O treinamento semanal consistia de trabalhos diários de segunda a sábado, de 40 - 50 minutos, divididos entre aquecimento, de aproximadamente 20 minutos com movimentos de aceleração ao trote e galope, seguido de paradas bruscas, recuos e movimentos em círculos. Posteriormente, os animais realizavam percursos ao trote e galope por mais 20-30 minutos.

Para a presente pesquisa, os equinos foram submetidos a uma prova de três tambores, que consiste em contornar os referidos obstáculos, distribuídos ao longo da pista, no menor tempo possível. Os tambores foram distribuídos de forma triangular, com uma distância de 27,5 m entre o primeiro e segundo tambores, e uma distância de $32 \mathrm{~m}$ entre os demais, conforme recomenda a Associação Brasileira dos Criadores de Cavalos Quarto de Milha (ABQM).

Foram quatro momentos de avaliação de cada um dos animais, sendo assim caracterizados: antes - T0 (imediatamente antes do exercício físico, com o animal ainda na baia, em repouso), T1 (num período máximo de cinco minutos após a realização da atividade física), T2 (30 minutos após a realização da atividade física) e T3 (duas horas após a realização da atividade física). Todas as atividades foram realizadas no período da manhã (entre 6h:00 e 11h:00), quando também foram registradas as características da pista, temperatura ambiente e umidade relativa do ar local. Foram usados quatro cavaleiros com peso corpóreo aproximado de $75 \mathrm{~kg}$ e altura de $1,75 \mathrm{~m}$.

As amostras de sangue foram obtidas, após antissepsia local, por meio de venopunção da jugular com agulhas descartáveis ( $25 \mathrm{~mm}$ x $0,8 \mathrm{~mm}$ ), utilizando-se sistema a pressão negativa, em tubos de plástico contendo anticoagulante EDTA com capacidade de $4 \mathrm{~mL}$, para determinação do VCM e do RDW por meio de analisador hematológico (Coulter HmX Hematology Analyzer, Beckman Coulter, Brea, CA, USA). Todas as amostras foram transportadas sob refrigeração ao Laboratório Clínico do Centro de Diagnóstico Veterinário (CDV, Vitória-ES), onde foram imediatamente processadas.

A análise dos resultados foi realizada utilizando-se o programa estatístico computadorizado GraphPad InStat, versão 3.0 (GraphPad, La Jolla, CA, USA). Os dados foram avaliados quanto à normalidade pelo teste de Kolmogorov-Smirnov, seguido de análise de variância de medidas repetidas (ANOVA) e comparação entre médias (teste de Tukey) com nível de significância de 5\%, visando verificar a influência do exercício físico sobre as concentrações das variáveis estudadas (VCM e RDW). Adicionalmente, foi feita a comparações dos valores registrados entre machos e fêmeas pelo teste-t, com nível de significância de 5\%. 


\section{Resultados}

Nos exames clínicos realizados para seleção dos equinos usados na presente pesquisa, os valores médios registrados foram: frequência cardíaca de $37 \mathrm{bpm}$, frequência respiratória de $21 \mathrm{mpm}$, motilidade intestinal presente à auscultação, mucosas róseas e temperatura retal de $37,4^{\circ} \mathrm{C} . \mathrm{Na}$ avaliação do hemograma, os valores médios encontrados foram de $7,74 \times 10^{6} / \mu \mathrm{L}$ para número de eritrócitos, 12,74 g/dL para concentração de hemoglobina, 36,0\% para volume globular e 9391,3 leucócitos/ $\mu \mathrm{L}$.

O experimento foi realizado ao longo de cinco dias, com cada equino sendo trabalhado uma única vez. As características do clima local são típicas de regiões tropicais, com altas temperaturas $\left(26,5 \pm 2,8^{\circ} \mathrm{C}\right)$ e alta umidade $(67,5 \pm 9,5 \%)$. A pista de areia encontrava-se seca. Segundo os cavaleiros, os animais responderam bem à atividade física executada, não demonstrando desconforto durante a realização da mesma.

Na Tabela 1 estão apresentados os valores médios e desvios-padrão para os valores de VCM e RDW, além dos valores de $p$ obtidos na análise de variância. É possível observar que o exercício imposto elevou significativamente somente o VCM nas fêmeas e machos estudados. Na comparação dos valores registrados para VCM e RDW pelo teste-t entre machos e fêmeas não foram encontradas diferenças significativas com valores de $\mathrm{p}>0,05$.

Tabela 1. Valores médios e desvios-padrão de volume corpuscular médio (VCM) e amplitude de distribuição do tamanho dos eritrócitos (RDW) nos equinos da raça Quarto de Milha, em uma sessão de exercício físico (prova de três tambores), nos momentos T0, T1, T2 e T3

\begin{tabular}{lllllll}
\hline & Sexo & T0 & T1 & T2 & T3 & $p$ \\
\hline VCM (fl) & Machos & $46,5 \pm 2,0^{\mathrm{a}}$ & $47,6 \pm 2,2^{\mathrm{b}}$ & $46,6 \pm 1,8^{\mathrm{a}}$ & $46,7 \pm 1,9^{\mathrm{a}}$ & $<0,0001$ \\
& Fêmeas & $46,3 \pm 0,6^{\mathrm{a}}$ & $47,7 \pm 0,9^{\mathrm{b}}$ & $46,7 \pm 0,8^{\mathrm{a}}$ & $46,4 \pm 0,8^{\mathrm{a}}$ & 0,0014 \\
RDW (\%) & Machos & $28,8 \pm 8,1^{\mathrm{a}}$ & $30,8 \pm 9,5^{\mathrm{a}}$ & $28,2 \pm 8,1^{\mathrm{a}}$ & $26,6 \pm 8,0^{\mathrm{a}}$ & 0,1295 \\
& Fêmeas & $28,0 \pm 7,3^{\mathrm{a}}$ & $27,1 \pm 8,1^{\mathrm{a}}$ & $28,1 \pm 8,1^{\mathrm{a}}$ & $28,6 \pm 7,2^{\mathrm{a}}$ & 0,8967 \\
\hline Nota: Letras minúsculas diferentes na mesma linha denotam diferença estatística significativa entre as medias (p \\
$<0,05$ ) obtida pelo teste de Tukey. T0 (antes da atividade física), T1 (num período de até no máximo cinco minutos \\
após o término da atividade física), T2 (30 minutos após o término da atividade fisica) e T3 (duas horas após o \\
término da atividade física).
\end{tabular}

\section{Discussão}

A avaliação clínica feita para selecionar os animais demonstra valores dentro da normalidade segundo Malikides et al. ${ }^{(12)}$.

Na presente pesquisa, o VCM registrado em todos os tempos de avaliação encontrava-se dentro da faixa de normalidade para a espécie equina, como descrito por Malikides et al. ${ }^{(12)}$. Os resultados foram similares aos relatos envolvendo equinos das raças Árabe ${ }^{(9,18,20)}$ e Quarto de Milha ${ }^{(21)}$, porém superiores aos relatos de Balarin et al. ${ }^{(10)}$ para equinos da raça PSI, de Masini et al. ${ }^{(24)}$ para equinos da raça Haflinger, e de Melo et al.(2) para animais da raça Mangalarga Marchador. Todos os autores supracitados fizeram suas pesquisas em animais atletas, informação que, segundo Lippi et al. ${ }^{(22)}$, deve ser considerada visto que os valores podem ser influenciados pela prática de atividade física. 
Já para RDW, os valores registrados nos Quarto de Milha na prova de três tambores, em todos os momentos de avaliação, foram similares aos descritos por Balarin et al. ${ }^{(10)}$, que avaliaram PSI no exercício e treinamento e encontraram resultados bem superiores aos demais relatos ${ }^{(2,11,16,21,24)}$. Os valores encontrados foram ainda superiores à descrição de Radin et al. ${ }^{(14)}$, que descreveram aumento significativo nos valores de RDW nos grupos com severa hemorragia e hemólise. Em sua pesquisa, Balarin et al. ${ }^{(10)}$ justificaram tanto as oscilações em VCM quanto em RDW como decorrentes das diferentes raças e protocolos laboratoriais, ressaltando a importância do estabelecimento de valores de referência para cada região e espécie / raça animal.

Conforme já mencionado, Lippi et al. ${ }^{(22)}$ destacaram que, dentre os diversos fatores influenciando variáveis hematimétricas, como VCM e RDW, estão se as amostras são de atletas em atividade ou não e o tipo e intensidade do esporte praticado. Corroborando com tal afirmativa, Miranda et al. ${ }^{(21)}$ destacaram a importância de se avaliar equinos nas diferentes modalidades atléticas. $\mathrm{O}$ aumento significativo registrado para VCM no momento T1 indicou que houve uma macrocitose eritrocitária, ou seja, a prova de três tambores levou ao aumento no tamanho dos eritrócitos circulantes, com retorno aos valores pré-exercício já em T2. Similarmente, Geor et al. ${ }^{(17)}$, trabalhando com PSI em velocidade de $13 \mathrm{~m} / \mathrm{s}$, Ferraz et al. ${ }^{(18)}$, trabalhando com Árabes submetidos à exercício progressivo em esteira, e Masini et al. ${ }^{(24)}$, exercitando equinos da raça Haflinger também na esteira, registraram a influência do exercício físico imposto sobre o VCM. Segundo Ferraz et al. ${ }^{(18)}$, na etapa de esforço máximo, há um aumento gradativo das variáveis hematológicas, dentre elas o VCM, devido à contração esplênica esforço-dependente, proporcional à intensidade do exercício físico. Esse efeito teria como principal benefício o aumento do transporte de oxigênio para o músculo esquelético. Por outro lado, Muñoz et al..$^{(9)}$ observaram uma redução do VCM nos equinos da raça Anglo-Árabe estudados e associaram tal achado à liberação de células menores e mais velhas por parte do baço ${ }^{(5)}$.

Diferentemente da presente pesquisa e dos trabalhos supracitados, Balarin et al. ${ }^{(10)}$, Machado ${ }^{(20)}$ e Miranda et al. ${ }^{(21)}$ observaram que o VCM não foi influenciado pelo exercício físico imposto em suas pesquisas. Miranda et al. ${ }^{(21)}$ sugeriram que a prova de team penning teria levado a uma liberação mínima e insuficiente por parte do baço. Já segundo Machado ${ }^{(20)}$, o VCM não variou devido à sobreposição de dois eventos, a liberação de células velhas e pequenas pelo baço e a tumefação eritrocitária. É possível sugerir que, nos animais usados na presente pesquisa, o VCM aumentou em consequência dessa tumefação dos eritrócitos. O exercício físico induz a queda do $\mathrm{pH}$, principalmente nos exercícios de alta intensidade e curta duração em que há predomínio de obtenção de energia por via anaeróbia ${ }^{(4)}$, tal como aqueles executados por animais da raça Quarto de Milha. Os eritrócitos desempenham função tampão, captando íons $\mathrm{H}^{+}$e $\mathrm{K}^{+}$e aumentando a entrada de água nas células ${ }^{(24)}$. Em sua pesquisa, Masini et al. ${ }^{(24)}$ testaram a teoria de que, além do baço, outros fatores influenciam a variação de VCM no exercício e comprovaram que o aumento do VCM aconteceu minutos depois do aumento do $\mathrm{VG}$, esse diretamente ligado à contração esplênica. $\mathrm{O}$ aumento significativo intraeritrocitário de íons potássio e cloretos associado com a redução da densidade celular também foi comprovado por outros autores ${ }^{(17)}$.

A prova de três tambores não gerou alteração do RDW, indicando que o aumento do tamanho eritrocitário aconteceu de forma homogênea, semelhante à pesquisa de Miranda et al. ${ }^{(21)}$. Diferentemente, em todas as demais citações ${ }^{(10,20,24)}$, os diferentes tipos de exercício levaram ao aumento no número de eritrócitos com anisocitose, com os autores correlacionando tal achado com a contração esplênica. Conforme descrito anteriormente, algumas citações associaram o aumento no RDW com o aumento do VCM, mas outras não, sugerindo que tais variáveis podem ser influenciadas por diversos fatores, dentre os quais raça e tipo de exercício ${ }^{(22)}$. É crucial que essas informações sejam bem caracterizadas para que seja feita a correta interpretação dos dados para fins diagnósticos e no uso de tais ferramentas no acompanhamento de programas de treinamento, bem como na avaliação do condicionamento atlético de equinos, associando os resultados com variáveis mais sensíveis, tais como frequência cardíaca e lactato plasmático.

Kowal et al. ${ }^{(3)}$ citaram que a normalização das variáveis hematimétricas tende a acontecer 45 minutos após a atividade física, fato comprovado na presente pesquisa, quando 30 minutos após o término do exercício, o VCM e RDW atingiram os valores registrados previamente ao teste. 


\section{Conclusão}

Os resultados da presente pesquisa permitem concluir que o exercício físico imposto (prova de três tambores) levou à macrocitose homogênea dos eritrócitos nos equinos da raça Quarto de Milha estudados. A recuperação dos valores pré-exercício aconteceram já aos 30 minutos após o término da atividade física, sugerindo que os animais estavam condicionados ao nível de atividade física imposta.

\section{Referências}

1. Tyler-McGowan CM, Golland LC, Evans DL, Hodgson DR, Rose RJ. Haematological biochemical responses to training and overtraining. Equine Veterinary Journal. 1999;30(suppl):621-625.

2. Melo SKM, Lira LB, Almeida TLAC, Rego EW, Manso HECCC, Manso Filho HC. Índices hematimétricos e bioquímica sanguínea no cavalo de cavalgada em condições tropicais. Ciência Animal Brasileira. 2013;14(2):208-215. Portuguese.

3. Kowal RJ, Almosny NRP, Summa R, Cury LJ. Avaliação dos valores hematológicos em cavalos (Equus caballus) da raça Puro-Sangue-Inglês (PSI) submetidos a teste de esforço em esteir ergométrica. Revista Brasileira de Ciência Veterinária. 2006;13(1):25-31. Portuguese.

4. Hanzawa K, Watanabe S. Changes in osmotic fragility of erythrocytes during exercise in athletic horses. Journal of Equine Science. 2000;11(3):51-61.

5. Persson S. On blood volume and working capacity in horses. Studies of methodology and physiological and pathological variations. Acta Veterinaria Scandinavica. 1967;19(9):189.

6. Lekeux P, Art T, Linden A, Desmecht D, Amory H. Heart rate, hematological and serum biochemical responses to show jumping. In: Persson SGB, Lindholm A, Jeffcott LB (Eds.). Equine Exercise Physiology. Davis: ICEEP Publications; 1991. p.385-390.

7. McKeever KH, Hinchcliff KW, Reed SM, Robertin JT. Role of decreased plasma volume in haematocrit alterations during incremental treadmill exercise in horses. American Journal of Physiology. 1993;265(2):404408.

8. Santos VP. Variações hemato-bioquímicas em eqüinos de salto submetidos a diferentes protocolos de exercício físico. Dissertação de Mestrado em Ciências Veterinárias, Universidade Federal do Rio Grande do Sul, Porto Alegre, 2006. 94p. Disponível em: http://www.ufrgs.br/lacvet/restrito/pdf/dm_vps.pdf

9. Muñoz A, Riber C, Trigo P, Castejón F. Erythrocyte indices in relation to hydration and electrolytes in horses performing exercises of different intensity. Compendium on Clinical Pathology. 2008;17(4):213-220.

10. Balarin MRS, Lopes RS, Kohayagawa A, Laposy CB, Fonteque JH. Valores da amplitude de distribuição do tamanho dos eritrócitos (RDW) em equinos Puro Sangue Inglês (PSI) submetidos a exercícios de diferentes intensidades. Brazilian Journal of Veterinary Research and Animal Science. 2006;43(5):637-641. Portuguese.

11. Balarin MRS, Fonteque JH, Souza C, Saito ME, Kohayagawa A, Lopes RS. Valores da amplitude de distribuição do tamanho dos eritrócitos (RDW - Red Cell Distribution Width) em equinos da raça Puro Sangue Inglês (PSI) de ambos os sexos de 12 a 24 meses de idade. Semina: Ciências Agrárias. 2001;22(2):135-137.

12. Malikides N, Hodgson DR, Rose RJ. Hemolymphatic System. In: Rose RJ, Hodgson DR. Manual of Equine Practice. 2nd ed. Philadelphia: WB Saunders; 2000. p. 451-474. 
13. Easley JR. Erythrogram and red cell distribution of equidae with experimentally induced anemia. American Journal of veterinary Research. 1985;46(11):2378-2384.

14. Radin MJ, Eubank MC, Weiser MG. Eletronic measurement of erythrocyte volume and volume heterogeneity in horses during erythrocyte regeneration associate with experimental anemias. Veterinary Pathology. 1986;23(6):656-660.

15. Muñoz A, Castejon FM, Rubio MD, Vivo R, Aguera EI, Escribano BM, Santisteban R. How erythrocyte and plasma lactate concentrations are related in Andalusian horses during an exercise test and recuperation. Journal of Equine Science. 1996;7(2):35-42.

16. Padalino B, Rubino G, Centoducati P, Petazzi F. Training versus overtraining: evaluation of two protocols. Journal of Equine Veterinary Science. 2007;27(1):28-31.

17. Geor RJ, Weiss DJ, Smith CM. Hemorheologic alterations induced by incremental treadmill exercise in thoroubreds. American Journal of Veterinary Research. 1994;55(6):854-861.

18. Ferraz GC, Teixeira-Neto AR, D'Angelis FHF, Lacerda-Neto JC, Queiroz Neto A. Alterações hematológicas e cardíacas em cavalos Árabes submetidos ao teste de esforço crescente em esteira rolante. Brazilian Journal of Veterinary Research and Animal Science. 2009;46(6):431-437. Portuguese.

19. McClay CB, Weiss DJ, Smith CM, Gordon B. Evaluation of hemorheologic variables as implications for exercise-induced pulmonary hemorrhage in racing thoroughbreds. American Journal of Veterinary Research. 1992;53(8):1380-1385.

20. Machado LP. Eritrograma, glutationa reduzida e superóxido dismutase eritrocitários e metahemoglobina em equinos da raça Árabe submetidos a exercício em esteira. Efeito da suplementação com vitamina E (DLAlfa-tocoferol). Dissertação de Mestrado em Medicina Veterinária, Universidade Estadual Paulista, Botucatu, 2006. 101p. Disponível em: http://base.repositorio.unesp.br/bitstream/handle/11449/89294/machado_lp_me_ botfmvz.pdf?sequence $=1 \&$ isAllowed=y . Portuguese.

21. Miranda RL, Mundim AV, Saquy ACS, Costa AS, Guimarães EC, Gonçalves FC, Silva FOC. Perfil hematológico de equinos submetidos à prova de Team Penning. Pesquisa Veterinária Brasileira. 2011;31(1):8186. Portuguese.

22. Lippi G, Salvagno GL, Danese E, Tarperi C, Guidi GC, Schena F. Variation of red blood cell distribution width and mean platelet volume after moderate endurance exercise. Advances in Hematology. 2014;43:349391.

23. Piccione G, Assenza A, Fazio F, Giudice E, Caola G. Different periodicities of some haematological parameters in exercise-loaded athletic horses and sedentary horses. Journal of Equine Science. 2001;12(1):1723.

24. Masini AP, Baragli P, Tedeschi D, Lubas G, Martelli F, Gavazza A, Sihieri C. Behaviour of mean erythrocyte volume during submaximal treadmill exercise in the horse. Comparative Haematology International. 2000;10(1):38-42. 